\title{
On the dual positive Schur property in Banach lattices
}

\author{
Witold Wnuk
}

Received: 7 August 2012 / Accepted: 23 August 2012 / Published online: 16 September 2012 (C) The Author(s) 2012. This article is published with open access at Springerlink.com

\begin{abstract}
The paper contains several characterizations of Banach lattices $E$ with the dual positive Schur property (i.e., $0 \leqslant f_{n} \stackrel{\sigma\left(E^{*}, E\right)}{\longrightarrow} 0$ implies $\left\|f_{n}\right\| \rightarrow 0$ ) and various examples of spaces having this property. We also investigate relationships between the dual positive Schur property, the positive Schur property, the positive Grothendieck property and the weak Dunford-Pettis property.
\end{abstract}

Keywords Schur property - Positive Schur property - Grothendieck property · Positive Grothendieck property

Mathematics Subject Classification (2000) $\quad$ 46B42 - 47B65 - 46B45 · 46E30

\section{Introduction}

Several types of the Schur property is considered in the theory of Banach lattices. Namely, a Banach lattice $E=(E,\|\cdot\|)$ has

- the Schur property, if $x_{n} \rightarrow 0$ weakly, then $\left\|x_{n}\right\| \rightarrow 0$,

- the positive Schur property, whenever $0 \leqslant x_{n} \rightarrow 0$ weakly implies $\left\|x_{n}\right\| \rightarrow 0$,

- the strong Schur property if there exists a number $K>0$ such that for all $\delta \in(0,2]$ every $\delta$-separated sequence in the unit ball contains a subsequence $K \delta$-equivalent to the standard $\ell^{1}$-basis,

This research was partially supported by The National Center of Science, Poland, Grant no. N N 201 605340 .

W. Wnuk $(\bowtie)$

Faculty of Mathematics and Computer Science, A. Mickiewicz University,

Umultowska 87, 61-614 Poznan, Poland

e-mail:wnukwit@amu.edu.pl 
- the dual positive Schur property, if $0 \leqslant f_{n} \rightarrow 0$ in the weak* topology, then $\left\|f_{n}\right\| \rightarrow 0$.

Results concerning the first three Schur type properties are scattered in a literature but the most essential characterizations, descriptions, examples etc. one can find in $[4,10,16,25,30,33]$. The present paper is devoted to the fourth property which was introduced in [6]. We present several characterizations of the property (Sect. 2) and examples of Banach lattices having the dual positive Schur property (Sect. 4). Our terminology and notations are standard-we follow by $[2,3,23,38]$.

Investigating the dual positive Schur property we easily find its relationships with the Grothendieck and the positive Grothendieck properties. Let us recall that a Banach lattice $E$ has

- the Grothendieck property if weak* null sequences in $E^{*}$ are weak null,

- the positive Grothendieck property if weak* null sequences with positive terms are weak null.

Results concerning the Grothendieck property are presented in [23, Section 5.3] and in [26] (see also [12] where classical function spaces having the Grothendieck property are investigated).

In the sequel we will use notations $E \in$ (SP), $E \in$ (PSP), $E \in$ (SSP), $E \in$ (DPSP), $E \in(\mathrm{GP}), E \in(\mathrm{PGP})$ to express that a Banach lattice $E$ has a suitable Schur or Grothendieck property. The "positive" properties are essentially weaker than "nonpositive". Indeed, $L^{1}(\mu) \in(\mathrm{PSP})$ but $L^{1}(\mu) \in(\mathrm{SP})$ if and only if $\mu$ is purely atomic. The space $c$ of convergent sequences has the positive Grothendieck property (there even holds $c \in$ (DPSP) because every positive functional on $c$ attends its norm at the sequence $(1,1,1, \ldots))$ but $c \notin(\mathrm{GP})$ because nonreflexive spaces having the Grothendieck property are not separable (see [23, Proposition 5.3.10]). Moreover, if $c_{0}$ is considered with a norm $\left\|\left(a_{k}\right)\right\|_{n}=\sup _{|F| \leqslant n} \sum_{n \in F}\left|a_{n}\right|$ and $E=\left(\oplus\left(c_{0},\|\cdot\|_{n}\right)\right)_{c_{0}}$, then $E^{*} \in(\mathrm{SP})$ but $E^{*} \notin(\mathrm{SSP})$ (this example was found by Knaust and Odell-see [33] for details). On the other hand (PSP) and (SP) coincide in the class of discrete Banach lattices (see [30, p. 19]) and (PGP) is equivalent to (GP) for Banach lattices having the interpolation property (see [23, Theorem 5.3.13]).

The lattice operations are discontinuous in the weak and weak* topologies in infinite dimensional spaces (see [2, Theorem 2.36]) which is a reason that "positive" properties are weaker than their "non-positive" prototypes. Sometimes the modulus is weakly sequentially continuous or sequentially weak* continuous. Indeed, since the weak convergence of sequences in spaces $C(K)$ means their norm boundedness and pointwise convergence then the modulus is weakly sequentially continuous in $C(K)$. The same holds in discrete Banach lattices with order continuous norms (see [23, Proposition 2.5.23]). Moreover, as it was noticed in [32, Theorem 6.6], the modulus is sequentially weak* continuous in the dual of a $\sigma$-Dedekind complete Banach lattice $E$ if and only if $E$ is discrete and its norm is order continuous. Below we present an alternative more elementary proof, based on the Schur property, of "if" part.

Proposition 1.1 If $E$ is a discrete Banach lattice with order continuous norm, then the operations $x \rightarrow|x|$ and $f \rightarrow|f|$ are sequentially $\sigma\left(E, E^{*}\right)$ continuous in $E$ and sequentially $\sigma\left(E^{*}, E\right)$ continuous in $E^{*}$, respectively. 
Proof Let $\left(e_{\alpha}\right)_{\alpha \in \mathcal{A}}$ be a complete disjoint system in $E$ consisting of discrete elements. Since the norm in $E$ is order continuous, then every $x \in E$ is of the form $x=\sum_{\alpha} t_{\alpha}(x) e_{\alpha}$ where numbers $t_{\alpha}(x)$ are uniquely determined and $\left\{\alpha: t_{\alpha}(x) \neq 0\right\}$ is at most countable. Functionals $f_{\alpha}$ defined by $f_{\alpha}(x)=t_{\alpha}(x)$ are homomorphisms, and so they are discrete in $E^{*}$. It is easy to see that $\left(f_{\alpha}\right)_{\alpha \in \mathcal{A}}$ is a complete disjoint system in $E^{*}$. According to [2, Theorem 1.75] for an arbitrary $f \in E^{*}$ there exists a unique family $\left(s_{\alpha}(f)\right)_{\alpha \in \mathcal{A}}$ of numbers such that $f=\left(\right.$ o) $\sum_{\alpha} s_{\alpha}(f) f_{\alpha}$, i.e. there exists a net $\left(g_{\Delta}\right) \subset E_{+}^{*}$ decreasing to zero, indexed by finite nonempty subsets of $\mathcal{A}$, satisfying the condition $\left|f-\sum_{\alpha \in \Delta} s_{\alpha}(f) f_{\alpha}\right| \leqslant g_{\Delta}$. Hence for every $x \in E$ there holds $\left|f(x)-\sum_{\alpha \in \Delta} s_{\alpha}(f) f_{\alpha}(x)\right| \leqslant g_{\Delta}(|x|) \downarrow 0$, i.e., $f(x)=\sum_{\alpha} s_{\alpha}(f) f_{\alpha}(x)$.

Suppose $g_{n} \rightarrow 0$ in the weak* topology and fix $x=\sum_{\alpha} t_{\alpha}(x) e_{\alpha} \in E$. Since the convergence is unconditional then for every $a=\left(a_{\alpha}\right) \in \ell^{\infty}(\mathcal{A})$ there exists $x_{a}=\sum_{\alpha} a_{\alpha} t_{\alpha}(x) e_{\alpha} \in E$. Clearly $g_{n}\left(x_{a}\right)=\sum_{\alpha} a_{\alpha} t_{\alpha}(x) s_{\alpha}\left(g_{n}\right)$, and so $c_{n}=\left(t_{\alpha}(x) s_{\alpha}\left(g_{n}\right)\right)_{\alpha} \in \ell^{1}(\mathcal{A})$. Moreover $g_{n}\left(x_{a}\right) \rightarrow 0$ for every $a$, i.e., $\left(c_{n}\right)$ is weakly null in $\ell^{1}(\mathcal{A})$. Hence $\left\|c_{n}\right\|=\sum_{\alpha}\left|t_{\alpha}(x) s_{\alpha}\left(g_{n}\right)\right| \rightarrow 0$ because $\ell^{1}(\mathcal{A}) \in(\mathrm{SP})$. Finally, for $x \in E_{+}$we obtain $\left|g_{n}\right|(x)=\sum_{\alpha}\left|s_{\alpha}\left(g_{n}\right)\right| t_{\alpha}(x)=\sum_{\alpha}\left|s_{\alpha}\left(g_{n}\right) t_{\alpha}(x)\right| \rightarrow 0$, i.e., $\left|g_{n}\right| \stackrel{\sigma\left(E^{*}, E\right)}{\longrightarrow} 0$.

Similar arguments work for the weak convergence. Assume $x_{m}=\sum_{\alpha} t_{\alpha}\left(x_{m}\right) e_{\alpha} \rightarrow$ 0 weakly in $E$. Without loss of generality we can assume $\mathcal{A}=\mathbb{N}$ because $\mathcal{A}_{0}=$ $\bigcup_{m=1}^{\infty}\left\{\alpha: t_{\alpha}\left(x_{m}\right) \neq 0\right\}$ is at most countable, $x_{m} \in B=\left\{e_{\alpha}: \alpha \in \mathcal{A}_{0}\right\}^{\mathrm{dd}}$, and we can restrict our considerations to the band $B$ where $\left(e_{\alpha}\right)_{\alpha \in \mathcal{A}_{0}}$ is an unconditional basis.

Fix $f \in E_{+}^{*}$. Since the expression of every $x \in E$ is unconditional, then the equality $f_{a}(x)=\sum_{n} a_{n} t_{n}(x) f\left(e_{n}\right)$ defines a continuous linear functional $f_{a}$ on $E$ for every $a=\left(a_{n}\right) \in \ell^{\infty}$. Therefore $\left(t_{n}(x) f\left(e_{n}\right)\right) \in \ell^{1}$. Hence elements $c_{m}=\left(t_{n}\left(x_{m}\right) f\left(e_{n}\right)\right)$ form a weak null sequence in $\ell^{1}$ because $\sum_{n} a_{n} t_{n}\left(x_{m}\right) f\left(e_{n}\right)=f_{a}\left(x_{m}\right) \rightarrow 0$ for every $a=\left(a_{n}\right) \in \ell^{\infty}$. Using the Schur property we obtain $f\left(\left|x_{m}\right|\right)=\sum_{n}\left|t_{n}\left(x_{m}\right)\right| f\left(e_{n}\right) \rightarrow$ 0 , i.e., $\left|x_{m}\right| \rightarrow 0$ in the weak topology.

Remark 1.2 It is worth to recall two results due to Chen and Wickstead related to Proposition 1.1.

1. If $E$ is a Banach lattice without discrete elements and the norm on $E$ is order continuous, then for every $x \in E_{+}$there exists a weak null sequence $\left(x_{n}\right)$ with $\left|x_{n}\right|=x$ for every $n$ (see [8, Theorem 2.2]).

2. If the dual $E^{*}$ does not contain discrete elements, then for every $f \in E_{+}^{*}$ there exists weak* null sequence $\left(f_{n}\right)$ such that $\left|f_{n}\right|=f$ for every $n$ (see $[9$, Theorem 3.1]).

It is easy to observe that the modulus preserves weak convergence of sequences with disjoint terms. Using [1, Lemma 1.6.1] and an idea from [31] we obtain the following result.

Proposition 1.3 If $\left|x_{n}\right| \wedge\left|x_{m}\right|=0$ and $x_{n} \rightarrow x$ weakly, then $x=0$ and $\left|x_{n}\right| \rightarrow 0$ weakly.

Proof Sequences $\left(x_{n}\right),\left(\left|x_{n}\right|\right)$ form unconditional basic sequences and $x \in \overline{\operatorname{span}}\left\{x_{n}\right.$ : $n \in \mathbb{N}\}$. If $\left(f_{n}\right)$ is the sequence of biorthogonal functionals associated to $\left(x_{n}\right)$ then $f_{k}(x)=\lim _{n \rightarrow \infty} f_{k}\left(x_{n}\right)=0$, and so $x=0$. The equality $\left|\sum_{k=n}^{m} t_{k}\right| x_{k}||=$ 
$\left|\sum_{k=n}^{m} t_{k} x_{k}\right|$ shows that the basic sequences are equivalent. Therefore $\overline{\operatorname{span}}\left\{x_{n}: n \in\right.$ $\mathbb{N}\}$ and $\overline{\operatorname{span}}\left\{\left|x_{n}\right|: n \in \mathbb{N}\right\}$ are isomorphic and there exists an isomorphism mapping $x_{n}$ onto $\left|x_{n}\right|$ (see [20] a remark after Definition 1.a.7). Finally $\left|x_{n}\right| \rightarrow 0$ weakly because the weak topology of a subspace is a restriction of the weak topology to this subspace.

An analogue of Proposition 1.3 is false for weak* convergence. An example presented below is based on an idea borrowed from [9]. Let $K$ be a compact space containing a sequence $\left(s_{n}\right)$ convergent to $s_{0}$ such that $s_{k} \neq s_{m}$ for $k \neq m$. Denote by $e_{n}$ the evaluation at point $s_{n}$. Define $F_{n} \in C(K)^{*}$ putting $F_{n}=e_{3 n-3}-e_{3 n-2}+e_{3 n-1}-e_{3 n}$ for even $n$ and $F_{n}=e_{3 n-2}-e_{3 n-1}$ for odd $n$. There holds $F_{m} \perp F_{j}$ for $m \neq j$ and $F_{n} \rightarrow 0 *$-weakly. On the other hand $\left|F_{2 n}\right| \rightarrow 4 e_{0}$ and $\left|F_{2 n-1}\right| \rightarrow 2 e_{0}$ in the weak* topology. Since $e_{0} \neq 0$, then the weak ${ }^{*}$ limit of $\left(\left|F_{n}\right|\right)$ does not exists.

Our example together with next proposition gives an alternative proof of the following known fact.

Let $K$ be an infinite quasi-Stonian compact space. A sequence $\left(s_{n}\right) \subset K$ is convergent if and only if it is eventually constant.

Proposition 1.4 Suppose that a Banach lattice $E$ is a $\sigma$-Dedekind complete. If $f_{n} \stackrel{\sigma\left(E^{*}, E\right)}{\longrightarrow} f$ and $\left|f_{m}\right| \wedge\left|f_{j}\right|=0$ for $m \neq j$, then $f=0$ and $\left|f_{n}\right| \stackrel{\sigma\left(E^{*}, E\right)}{\longrightarrow} 0$.

Proof Let a sequence $\left(f_{n}\right)$ consisting of disjoint elements be weak ${ }^{*}$ convergent to $f$. Choose $x \in E$ and $\varepsilon>0$. According to [3, Theorem 4.42] we can find $g \in E_{+}^{*}$ satisfying the condition $\left(\left|f_{n}\right|-g\right)^{+}(|x|) \leqslant \frac{\varepsilon}{2}$ for all $n$. Put $h_{n}=\left(\left|f_{n}\right|-g\right)^{+}$. Since $\left|f_{n}\right| \leqslant h_{n}+g$, then by Riesz decomposition property $\left|f_{n}\right|=u_{n}+v_{n}$ where $u_{n}, v_{n} \in E_{+}^{*}$ and $u_{n} \leqslant h_{n}, v_{n} \leqslant g$. Clearly $v_{n} \leqslant\left|f_{n}\right|$ and so $v_{m} \wedge v_{k}=0$. Hence $\sum_{n=1}^{m} v_{n}(|x|)=\left(\bigvee_{n=1}^{m} v_{n}\right)(|x|) \leqslant g(|x|)$ and we obtain $v_{n}(|x|)<\frac{\varepsilon}{2}$ for sufficiently large $n$. Therefore ||$f_{n}|(x)| \leqslant\left|f_{n}\right|(|x|)=u_{n}(|x|)+v_{n}(|x|) \leqslant h_{n}(|x|)+v_{n}(|x|)<\varepsilon$ for large $n$. We have just proved $\left|f_{n}\right| \rightarrow 0$ in the weak* topology what implies $f_{n} \stackrel{\sigma\left(E^{*}, E\right)}{\longrightarrow}$ 0, i.e., $f=0$.

Remark 1.5 As we showed before, Proposition 1.4 is false if we reject the assumption of the $\sigma$-Dedekind completeness. On the other hand there exist non $\sigma$-Dedekind complete Banach lattices for which the conclusion of Proposition 1.4 remains true. Indeed, it is well known that $\ell^{\infty} \in(\mathrm{GP})$ and that the Grothendieck property is preserved by quotients. Hence the conclusion of Proposition 1.4 holds for $E=\ell^{\infty} / c_{0}$ due to Proposition 1.3 but $\ell^{\infty} / c_{0}$ is not $\sigma$-Dedekind complete.

\section{The dual positive Schur property and the positive Grothendieck property}

It is clear that every (linear and continuous) operator $T: E \rightarrow c_{0}$ is represented by some weak* null sequence $\left(f_{n}\right)$ and $T$ is weakly compact (compact) if and only if $f_{n} \rightarrow 0$ weakly ( $f_{n} \rightarrow 0$ in norm). Therefore the following equivalences are obvious.

$E \in(\mathrm{PGP})$ if and only if every positive operator $T: E \rightarrow c_{0}$ is weakly compact,

$E \in$ (DPSP) if and only if every positive operator $T: E \rightarrow c_{0}$ is compact.

Combining the open mapping theorem with the above operator characterizations we get that positive surjections preserve the dual positive Schur property and the positive 
Grothendieck property. In particular positively complemented Riesz subspaces and quotients (by closed ideals) of spaces with the dual positive Schur property (positive Grothendieck property) also have the property. It is also completely clear that

$$
E \in(\mathrm{DPSP}) \text { if and only if } E^{*} \in(\mathrm{PSP}) \text { and } E \in(\mathrm{PGP}) \text {. }
$$

The last equivalence has several simple consequences.

Proposition 2.1 Let a Banach lattice $E \in$ (DPSP). Then

(a) The dual $E^{*}$ has order continuous norm.

(b) The norm on $E$ is not order continuous when $E$ is infinite dimensional.

(c) $E^{*} \notin$ (DPSP) whenever $\operatorname{dim} E=\infty$.

(d) If $E$ is additionally $\sigma$-Dedekind complete and infinite dimensional, then $E$ is non separable and $E^{*} \in(\mathrm{PSP})$ but $E^{*} \notin(\mathrm{SP})$ (in particular, $E^{*}$ is not discrete).

Proof (a) The positive Schur property implies the order continuity of a norm.

(b) It is enough to apply the following characterization: a norm in $E$ is order continuous if and only if norm bounded disjoint positive functionals on $E$ are weak* convergent to zero (see [32, Theorem 2.2]).

(c) is obvious as in immediate consequence of (a) and (b).

(d) If $E$ were separable then the norm on $E$ would be order continuous which contradicts (b). Similarly, supposing $E^{*} \in(\mathrm{SP})$ we obtain $E$ does not contain any subspace isomorphic to $\ell^{1}$ (see [30, Theorem 1]). According to [32, Theorem 4.7] both $E$ and $E^{*}$ have order continuous norms which is impossible by (b). The dual $E^{*}$ can not be discrete because for discrete spaces the (PSP) and (SP) coincide.

A result of Dodds-Fremlin ([11, Corollary 2.7]) will be useful for our further considerations. We remind the reader of it below (an elegant and short proof may be found in [13, Proposition 4]).

Theorem 2.2 Let $E$ be a Banach lattice. A sequence $\left(f_{n}\right) \subset E^{*}$ is norm convergent to zero if and only if $\left|f_{n}\right| \rightarrow 0$ in the weak* topology and $f_{n}\left(x_{n}\right) \rightarrow 0$ for every norm bounded disjoint sequence $\left(x_{n}\right) \subset E$.

Our next result shows that we can restrict sequences appearing in the definition of the dual positive Schur property to disjoint sequences. It is useful when we want to verify if a concrete Banach lattice has the dual positive Schur property.

Proposition 2.3 A Banach lattice E has the dual positive Schur property if and only if an arbitrary sequence of positive disjoint functionals weak ${ }^{*}$ convergent to zero is norm null.

Proof Let $E_{+}^{*} \ni f_{n} \rightarrow 0$ in the weak* topology and let disjoint terms of a sequence $\left(x_{n}\right)$ belong to positive part of the unit ball of $E$. According to Theorem 2.2 it is sufficient to show $f_{n}\left(x_{n}\right) \rightarrow 0$. Suppose that our conjecture is false. Passing to a subsequence if necessary we can assume $f_{n}\left(x_{n}\right)>\varepsilon>0$ for all $n$. Applying [32, Proposition 0.3.11] we find $g_{n} \in E_{+}^{*}$ such that $g_{n} \wedge g_{m}=0, g_{n} \leqslant f_{n}$ and $g_{n}\left(x_{n}\right)=$ $f_{n}\left(x_{n}\right)$. Therefore $\left(g_{n}\right)$ is weak* null, and it should be $\left\|g_{n}\right\| \rightarrow 0$ but $\varepsilon<f_{n}\left(x_{n}\right)=$ $g_{n}\left(x_{n}\right) \leqslant\left\|g_{n}\right\|$, a contradiction. 
It is worth to note that the condition

$$
(+) f_{k} \wedge f_{m}=0 \text { and } f_{n} \stackrel{\sigma\left(E^{*}, E\right)}{\longrightarrow} 0 \text { implies } f_{n} \stackrel{\sigma\left(E^{*}, E^{* *}\right)}{\longrightarrow} 0
$$

does not characterize the positive Grothendieck property. Indeed, every AL-space $E$ satisfy the condition (+) because weak* convergent sequences $\left(f_{n}\right)$ in $E^{*}$ are order bounded, and so they are weakly convergent whenever $f_{k} \wedge f_{m}=0$. On the other hand none infinite dimensional AL-space $E \in(\mathrm{PGP})$. If it were true that $E \in$ (PGP) there would be $\ell^{1} \in$ (PGP) because $\ell^{1}$ is positively complemented in $E$ but for functionals $f_{n} \in\left(\ell^{1}\right)^{*}$ represented by the characteristic functions of sets $\mathbb{N} \backslash\{1, \ldots, n\}$ there holds $f_{n} \rightarrow 0$ in the weak* topology and $F\left(f_{n}\right)=1$, where $F$ is the Banach limit, i.e., $\left(f_{n}\right)$ is not weak null.

The authors of [6] noticed that the dual positive Schur property is related to the so called bi-sequence property. We say that a Banach lattice E satisfies the bi-sequence property if $f_{n}\left(x_{n}\right) \rightarrow 0$ for every weak null sequence $\left(x_{n}\right) \subset E_{+}$consisting of disjoint elements and every weak ${ }^{*}$ null sequence $\left(f_{n}\right) \subset E_{+}^{*}$. Our succeeding result shows conditions equivalent to the bi-sequence property.

Proposition 2.4 For a Banach lattice $E$ the following statements are equivalent.

(a) For arbitrary sequences $\left(x_{n}\right) \subset E, \quad\left(f_{n}\right) \subset E^{*}$ such that $x_{k} \wedge x_{m}=0$, $f_{k} \wedge f_{m}=0, x_{n} \stackrel{\sigma\left(E, E^{*}\right)}{\longrightarrow} 0, f_{n} \stackrel{\sigma\left(E^{*}, E\right)}{\longrightarrow} 0$ there holds $f_{n}\left(x_{n}\right) \rightarrow 0$.

(b) E has the bi-sequence property.

(c) For all sequences $\left(x_{n}\right) \subset E_{+},\left(f_{n}\right) \subset E^{*}$ such that $f_{k} \wedge f_{m}=0, x_{n} \stackrel{\sigma\left(E, E^{*}\right)}{\longrightarrow} 0$, $f_{n} \stackrel{\sigma\left(E^{*}, E\right)}{\longrightarrow} 0$ there holds $f_{n}\left(x_{n}\right) \rightarrow 0$.

(d) For all sequences $\left(x_{n}\right) \subset E_{+},\left(f_{n}\right) \subset E_{+}^{*}$ such that $x_{n} \stackrel{\sigma\left(E, E^{*}\right)}{\longrightarrow} 0, f_{n} \stackrel{\sigma\left(E^{*}, E\right)}{\longrightarrow} 0$ there holds $f_{n}\left(x_{n}\right) \rightarrow 0$.

Proof (a) $\Rightarrow$ (b) It is enough to repeat the proof of Proposition 2.3.

(b) $\Rightarrow$ (d) Let $\left(x_{n}\right) \subset E_{+},\left(f_{n}\right) \subset E_{+}^{*}$ be weak null and weak* null respectively. Since $X=\left\{x_{n}: n \in \mathbb{N}\right\} \cup\{0\}$ is weakly compact, then according to [3, Theorem 4.34] $v_{n} \rightarrow 0$ weakly for every sequence $\left(v_{n}\right) \subset$ sol $X$ consisting of disjoint elements. The bi-sequence property implies $\sup _{k}\left|f_{k}\left(v_{n}\right)\right| \rightarrow 0$. Hence an operator $T: E \rightarrow$ $c_{0}$ defined by $T(x)=\left(f_{n}(x)\right)$ satisfies a condition $\left\|T\left(v_{n}\right)\right\| \rightarrow 0$ for sequences $\left(v_{n}\right) \subset$ sol $X$ of disjoint elements. Fix $\varepsilon>0$. Applying [3, Theorem 4.36] we can find $u_{\varepsilon} \in E_{+}$such that $\sup _{k}\left|f_{k}\left(\left(x_{n}-u_{\varepsilon}\right)^{+}\right)\right|=\left\|T\left(\left(x_{n}-u_{\varepsilon}\right)^{+}\right)\right\|<\frac{\varepsilon}{2}$ for all $n$. Moreover $f_{n}\left(u_{\varepsilon}\right)<\frac{\varepsilon}{2}$ for sufficiently large $n$. Finally $0 \leqslant f_{n}\left(x_{n}\right) \leqslant f_{n}\left(\left(x_{n}-u_{\varepsilon}\right)^{+}\right)+f_{n}\left(u_{\varepsilon}\right) \leqslant$ $\sup _{k}\left|f_{k}\left(\left(x_{n}-u_{\varepsilon}\right)^{+}\right)\right|-f_{n}\left(u_{\varepsilon}\right)<\varepsilon$ for large $n$, i.e., $f_{n}\left(x_{n}\right) \rightarrow 0$.

(d) $\Rightarrow$ (c) $\Rightarrow$ (a) Obvious.

Below we formulate a few simple characterizations of the dual positive Schur property. They show the property is related to the weak Dunford-Pettis property considered in [26, Section 9], [5,31]. Let us recall that a Banach lattice E has the weak DunfordPettis property (shortly $E \in(\mathrm{wDPP})$ ) if every weakly compact operator on $E$ maps weak null disjoint sequences onto norm convergent. As it was shown in [5, Theorem 2.2] the condition $E \in(\mathrm{wDPP})$ means exactly that weakly compact operators on $E$ transform weak null positive sequences onto norm convergent. 
Proposition 2.5 For a Banach lattice $E$ the following statements are equivalent.

(a) $E \in$ (DPSP).

(b) $E \in(\mathrm{PGP})$ and $E^{*} \in(\mathrm{PSP})$.

(c) $E \in(\mathrm{PGP})$ and $E \in(\mathrm{wDPP})$.

(d) $E^{*}$ has order continuous norm and $E$ has the bi-sequence property.

Remark 2.6 The equivalence (a) $\Leftrightarrow$ (d) was proved in [6].

Proof (a) $\Rightarrow$ (b) Obvious.

(b) $\Rightarrow$ (c) By [31, Theorem 4] a condition $E^{*} \in(\mathrm{PSP})$ implies $E \in$ (wDPP).

(c) $\Rightarrow$ (d) Since the positive Grothendieck property is inherited by positively complemented Riesz subspaces and, as we have already noticed, $\ell^{1} \notin$ (PGP), then our the assumption $E \in$ (PGP) implies $E$ does not contain any Riesz subspace order isomorphic to $\ell^{1}$, i.e., the dual norm is order continuous (see [32, Theorem 3.2]). Suppose that $x_{m} \wedge x_{k}=0, x_{n} \rightarrow 0$ weakly, $0 \leqslant f_{n} \rightarrow 0$ in the topology $\sigma\left(E^{*}, E\right)$. The positive Grothendieck property implies the operator $T: E \rightarrow c_{0}$ given by the equality $T(x)$ $=\left(f_{n}(x)\right)$ is weakly compact. Therefore $0 \leqslant f_{n}\left(x_{n}\right) \leqslant \sup _{k}\left|f_{k}\left(x_{n}\right)\right|=\left\|T\left(x_{n}\right)\right\| \rightarrow$ 0 by the weak Dunford-Pettis property, i.e., $E$ has the bi-sequence property.

(d) $\Rightarrow$ (a) see [6] the proof of Proposition 3.4.

The dual positive Schur property can be also characterized by suitable properties of operators.

Proposition 2.7 For a Banach lattice E the following statements are equivalent.

(a) $E \in$ (DPSP).

(b) Every order weakly compact operator on E is M-weakly compact.

(c) Every positive weakly compact operator mapping E into a Banach lattice is semicompact.

(d) If $F$ is a discrete Banach lattice with order continuous norm, then every positive operator $T: E \rightarrow F$ is compact.

(e) Every positive operator $T: E \rightarrow c_{0}$ is compact.

Proof (a) $\Rightarrow$ (b) Since $E \in$ (PGP) then by [23, Theorem 5.3.13] order weakly compact operators on $E$ are weakly compact but weakly compact operators on $E$ are M-weakly compact because $E^{*} \in$ (PSP)-see [30, Theorem 8].

(b) $\Rightarrow$ (c) Apply [3, Theorem 5.72].

(c) $\Rightarrow$ (d) It is enough to notice that almost order bounded sets in discrete Banach lattices with order continuous norms are relatively compact.

(d) $\Rightarrow$ (e) $\Rightarrow$ (a) are obvious.

The statement (d) formulated in Proposition 2.7 motivates considerations of the following property of a Banach lattice $E$.

(*) If $F$ is a Banach lattice with order continuous norm and $T: E \rightarrow F$ is positive, then $T$ is compact.

Let us note that $\sigma$-Dedekind complete Banach lattices $E$ satisfying $(*)$ are finite dimensional. Indeed, condition $(*)$ implies $E$ does not contain any order isomorphic copy of $\ell^{\infty}$. On the contrary there would exist a positive projection $P: E \rightarrow \ell^{\infty}$. On account of [32, Theorem 6.5] we can choose a positive non compact operator 
$T: \ell^{\infty} \rightarrow L^{1}[0,1]$. Thus the composition $T \circ P$ is a non compact map from $E$ into $L^{1}[0,1]$, a contradiction. Since $E$ is $\sigma$-Dedekind complete and contains no copies of $\ell^{\infty}$ its norm is order continuous. Condition (*) implies the identity on $E$ is compact, i.e., $E$ is finite dimensional.

On the other hand there exist spaces $C(K)$ satisfying $(*)$ and we can characterize them.

Proposition 2.8 For an AM-space $E$ with an order unit the following statements are equivalent.

(a) $E^{*}$ is order isomorphic to $\ell^{1}(\Gamma)$ for some set $\Gamma$.

(b) E does not contain any closed subspace isomorphic (i.e., linearly homeomorphic) to $\ell^{1}$.

(c) E satisfies $(*)$.

(d) Every positive operator $T: E \rightarrow\left(\ell^{\infty}\right)^{*}$ is compact.

Proof (a) $\Rightarrow$ (b) Since $E^{*} \in$ (SP) then $E$ does not contain any copy of $\ell^{1}$ (see [30, Theorem 1]).

(b) $\Rightarrow$ (c) Suppose $F$ is a Banach lattice with the order continuous norm. Every positive operator $T: E \rightarrow F$ maps the unit ball, which is an order bounded set, into a weakly compact set in $F$ because order intervals in $F$ are weakly compact. The space $E$ has the Dunford-Pettis property, and so $T$ is a Dunford-Pettis operator but classes of Dunford-Pettis operators and compact operators defined on spaces without copies of $\ell^{1}$ are the same.

(c) $\Rightarrow$ (d) Obvious.

(d) $\Rightarrow$ (a) Using [32, Theorem 6.7] we obtain $E^{*}$ is a discrete AL-space, and so it is order isomorphic to some $\ell^{1}(\Gamma)$.

Remark 2.9 If $K$ is a countable compact space then $C(K)$ satisfies $(*)$ because $(C(K))^{*}$ is isomorphic to $\ell^{1}$ (see [1, p. 97 Remark 4.5.3]).

We should also mention a necessary condition for the dual positive Schur property which is due to Wójtowicz (see [35, Theorem 1]). Since the result is presented in [35] without proof we show it below with details.

Proposition 2.10 If a Banach lattice $E$ has the dual positive Schur property, then $E$ can not be positively and surjectively mapped onto any infinite dimensional Banach lattice with order continuous norm.

Proof Assume that an infinite dimensional Banach lattice $F$ possesses order continuous norm and $T: E \rightarrow F$ is a positive surjection. The adjoint operator $T^{*}$ is a positive isomorphic embedding by the closed range theorem (see [27, Theorem 11-3-4]). Since $F^{*}$ is infinite dimensional it contains a sequence $\left(g_{n}\right)$ of norm one positive disjoint element. It has to be $g_{n} \rightarrow 0$ in the weak* topology according to the order continuity of $F$ (see [32, Theorem 2.2]). Clearly $T^{*}\left(g_{n}\right) \neq 0$ by injectivity of $T^{*}$. Put $f_{n}=T^{*}\left(g_{n}\right) /\left\|T^{*}\left(g_{n}\right)\right\|$. There holds $0 \leqslant f_{n},\left\|f_{n}\right\|=1$ and $M=\inf \left\|T^{*}\left(g_{n}\right)\right\|>0$. Hence $\left|f_{n}(x)\right| \leqslant M^{-1}\left|g_{n}(T(x))\right|$ for every $x \in E$, i.e., $f_{n} \rightarrow 0$ in the weak* topology, which contradicts the dual positive Schur property of $E$. 
In the last section we will show that the theorem converse to Proposition 2.10 is false. We finish this part formulating some characterization of the positive Grothendieck property which is closely related to exercise 5.3.E2 in [23]. The following version of Räbiger's Lemma (see [26, Lemma 2.1]) will be useful as a tool of a proof.

Lemma 2.11 For a Banach lattice $E$ the following statements are equivalent.

(a) There does not exist any positive linear surjective operator from $E$ onto $c_{0}$.

(b) If $0 \leqslant f_{n} \rightarrow 0$ in the topology $\sigma\left(E^{*}, E\right)$, then $\left(f_{n}\right)$ is not equivalent to the unit vector basis in $\ell^{1}$.

We omit the proof of Lemma 2.11 because it is enough to repeat arguments used in [26].

Proposition 2.12 For a Banach lattice E the following statements are equivalent.

(a) $E \in(\mathrm{PGP})$.

(b) If $F$ is a non reflexive Banach lattice with order continuous norm, then none positive operator $T: E \rightarrow F$ is surjective.

(c) There does not exists any positive surjection $T: E \rightarrow c_{0}$.

Proof (a) $\Rightarrow$ (b) Suppose $F$ is a non reflexive Banach lattice whose norm is order continuous. The space $F$ contains an order isomorphic copy of $\ell^{1}$ or $c_{0}$ (see [32, Theorem 8.1]). Order copies of $\ell^{1}$ are positively complemented in every Banach lattice and order continuity of the norm implies that also order copies of $c_{0}$ are positively complemented in $F$ (see [32, Theorem 1.8]). If $T: E \rightarrow F$ were a positive surjection, then the composition of $T$ with a positive projection would be a positive surjection mapping $E$ onto $\ell^{1}$ or $c_{0}$ but positive surjective images of spaces with the positive Grothendieck property have the positive Grothendieck, a contradiction because $\ell^{1}, c_{0} \notin$ (PGP).

(b) $\Rightarrow$ (c) Obvious.

(c) $\Rightarrow$ (a) It is known that there exists a positive linear surjection from $\ell^{1}$ onto $c_{0}$ (see [23] exercise 2.1.E1), and so $E$ does not possess any positively complemented copy of $\ell^{1}$, i,e, the dual norm is order continuous which means the weak sequential completeness of $E^{*}$. Let $0 \leqslant f_{n} \stackrel{\sigma\left(E^{*}, E\right)}{\longrightarrow} 0$. According to Lemma 2.11 and the Rosenthal's $\ell^{1}$-theorem the sequence $\left(f_{n}\right)$ contains a weak Cauchy subsequence $\left(f_{n_{k}}\right)$, and so $\left(f_{n_{k}}\right)$ is weakly convergent. Hence $f_{n} \rightarrow 0$ in the weak topology.

\section{A remark on $\lambda$-sums of Banach lattices}

Let $E_{k}=\left(E_{k},\|\cdot\|\right), k \in \mathbb{N}$, be Banach lattices and let $\lambda=\left(\lambda,\|\cdot\|_{\lambda}\right)$ be a Banach lattice which is an ideal in $\mathbb{R}^{\mathbb{N}}$ containing all unit vectors. The space $\left(\oplus E_{k}\right)_{\lambda}$, called the $\lambda$-sum of spaces $E_{k}$, is defined as follows

$$
\left(\oplus E_{k}\right)_{\lambda}=\left\{\left(x_{k}\right) \in \prod_{k} E_{k}:\left(\left\|x_{k}\right\|_{k}\right) \in \lambda\right\} .
$$

The space $\left(\oplus E_{k}\right)_{\lambda}$ is a Banach lattice under the order induced from the product $\prod_{k} E_{k}$ and under the norm $\left\|\left(x_{k}\right)\right\|=\left\|\left(\left\|x_{k}\right\|_{k}\right)\right\|_{\lambda}$. It is well known that the space $\lambda_{n}$ of order continuous functionals on $\lambda$ (which are norm continuous because they are order 
bounded-see [3, Lemma 1.54]) can be identified with the Köthe dual $\lambda^{\times}=\left\{\left(b_{k}\right) \in\right.$ $\mathbb{R}^{\mathbb{N}}:\left(a_{k} b_{k}\right) \in \ell^{1}$ for every $\left.\left(a_{k}\right) \in \lambda\right\}$. The correspondence $\lambda_{n} \ni f \rightarrow\left(b_{k}\right) \in \lambda^{\times}$ is defined by $f\left(\left(a_{k}\right)\right)=\sum_{k=1}^{\infty} a_{k} b_{k}$ and $\lambda^{\times}$is considered with the norm $\left\|\left(b_{k}\right)\right\|=$ $\sup _{\left\|\left(a_{k}\right)\right\|_{\lambda} \leqslant 1}\left|\sum_{k} a_{k} b_{k}\right|$. The space $\lambda^{\times}$becomes a Banach lattice under the standard pointwise order. Since $\lambda^{\times}$contains unit vectors and it is complete then $\lambda^{\times}$contains sequences with strictly positive terms. For more details concerning Köthe duals we refer the reader to [17, Chapter 6, section 1] and [37, Chapter 15].

A description of the space of order continuous functionals on $\left(\oplus E_{k}\right)_{\lambda}$ is an aim of our present considerations. We will need an additional notation. Namely, if a sequence $\left(\sum_{k=1}^{n} x_{k}\right)$ is order convergent to $x$ we will write $x=(\mathrm{o}) \sum_{k=1}^{\infty} x_{k}$.

Let $E=\left(\oplus E_{k}\right)_{\lambda}$ and let $J_{k}: E_{k} \rightarrow E$ be an operator defined by $J_{k}(x)=$ $(0, \ldots, 0, x, 0, \ldots)$ where $x$ is located on $k$-th place. Clearly $J_{k}$ is an order isomorphism onto a band in $E$. The spaces of order continuous functionals on $E$ and $E_{k}$ will be denoted by $E_{n}$ and $\left(E_{k}\right)_{n}$ respectively.

Fix $f \in E_{n}$ and put $f_{k}=f \circ J_{k}$. Clearly $f_{k} \in\left(E_{k}\right)_{n}$. We claim $\left(\left\|f_{k}\right\|_{k}^{*}\right) \in \lambda^{\times}$ where $\|\cdot\|_{k}^{*}$ is the dual norm in $E_{k}^{*}$. Let all terms of a sequence $\left(b_{k}\right) \in \lambda^{\times}$be strictly positive. Choose $x_{k}$ in the unit ball of $E_{k}$ such that $\left\|f_{k}\right\|_{k}^{*}<\left|f_{k}\left(x_{k}\right)\right|+$ $b_{k}$ and let $\varepsilon_{k} \in\{-1,0,1\}$ be such that $\varepsilon_{k} f_{k}\left(x_{k}\right)=\left|f_{k}\left(x_{k}\right)\right|$. Since $\lambda$ is an ideal in $\mathbb{R}^{\mathbb{N}}$ then $\left(\left|a_{k}\right| \varepsilon_{k} x_{k}\right) \in E$ for an arbitrary $\left(a_{k}\right) \in \lambda$ and $f\left(\left(\left|a_{k}\right| \varepsilon_{k} x_{k}\right)\right)$ $=f\left((\mathrm{o}) \sum_{k=1}^{\infty} J_{k}\left(\left|a_{k}\right| \varepsilon_{k} x_{k}\right)\right)=\sum_{k=1}^{\infty}\left|a_{k}\right|\left|f_{k}\left(x_{k}\right)\right|$ by order continuity of $f$. Therefore $\sum_{k=1}^{\infty}\left|a_{k}\right|\left\|f_{k}\right\|_{k}^{*} \leqslant f\left(\left(\left|a_{k}\right| \varepsilon_{k} x_{k}\right)\right)+\sum_{k=1}^{\infty}\left|a_{k}\right| b_{k}<\infty$ and we are done. We have just proved that the equality $T(f)=\left(f_{k}\right)$ defines a linear operator mapping $E_{n}$ into $\left(\oplus\left(E_{k}\right)_{n}\right)_{\lambda \times}$.

Let us note that for every $0 \leqslant x \in E_{k}$ there holds $\left(|f| \circ J_{k}\right)(x)=$ $\sup _{|z| \leqslant J_{k}(x)}|f(z)|=\sup _{|y| \leqslant x} \mid f\left(J_{k}(y)|=| f \circ J_{k} \mid(x)\right.$, and so $T$ is a homomorphism. If $\left(x_{k}\right) \in E$, then $f\left(\left(x_{k}\right)\right)=f\left((0) \sum_{k=1}^{\infty} J_{k}\left(x_{k}\right)\right)=\sum_{k=1}^{\infty} f_{k}\left(x_{k}\right)$. The last observation implies injectivity of $T$. Moreover $T$ is a surjection. Indeed, fix $\left(f_{k}\right) \in\left(\oplus\left(E_{k}\right)_{n}\right)_{\lambda \times}$. For an arbitrary $x=\left(x_{k}\right) \in E$ the series $\sum_{k=1}^{\infty} f_{k}\left(x_{k}\right)$ is absolutely convergent and the formula $f(x)=\sum_{k=1}^{\infty} f_{k}\left(x_{k}\right)$ defines a linear functional on $E$. We claim $f \in E_{n}$. Assume $x_{\alpha}=\left(x_{\alpha}(k)\right) \downarrow_{\alpha} 0$ in $E$. Choose $\varepsilon>0$ and $\alpha_{0}$. Let $k_{0}$ be such that $\sum_{k=k_{0}+1}^{\infty}\left|f_{k}\left(x_{\alpha_{0}}(k)\right)\right| \leqslant \frac{\varepsilon}{2}$. Since $x_{\alpha}(k) \downarrow 0$ in $E_{k}$ and $f_{k} \in\left(E_{k}\right)_{n}$, then $\sum_{k=1}^{k_{0}}\left|f_{k}\left(x_{\alpha}(k)\right)\right|<\frac{\varepsilon}{2}$ for sufficiently large $\alpha \geqslant \alpha_{0}$. Hence $\left|f\left(x_{\alpha}\right)\right|<\varepsilon$ for large $\alpha$.

Our previous considerations lead us to the following result.

Proposition 3.1 If $E=\left(\oplus E_{k}\right)_{\lambda}$, then the space $E_{n}$ of order continuous functionals on $E$ is order isomorphic (and so linearly homeomorphic ) to $\left(\oplus\left(E_{k}\right)_{n}\right)_{\lambda \times}$.

The $\lambda$-sums allows us to produce examples of Banach lattices with various properties. For instance repeating arguments used in [30] we can prove that if $\lambda \in$ (SP), then $\left(\oplus E_{k}\right)_{\lambda} \in(\mathrm{SP})$ whenever all $E_{k}$ have the Schur property and $\left(\oplus E_{k}\right)_{\lambda} \in$ (PSP) when $E_{k} \in(\mathrm{PSP})$ for all $k$.

\section{Examples}

It is easy to check that $C(K) \in$ (DPSP) (compare [6]) for every compact space $K$ because positive functionals on $C(K)$ attend their norms on the constant function 
equals one. Using [23, Theorem 5.3.13] and remembering that $(C(K))^{*} \in$ (PSP), we obtain quickly a characterization of AM-spaces having the positive dual Schur property.

Proposition 4.1 For an AM-space E the following statements are equivalent.

(a) $E \in$ (DPSP).

(b) $E \in$ (PGP).

(c) E does not contain any positively complemented order copy of $c_{0}$.

If an AM-space $E \in$ (DPSP), then also its even duals have the dual positive Schur property because they are AM-spaces with a strong unit. Below we show that many Musielak-Orlicz spaces have the dual positive Schur property.

Let $(T, \Sigma, \mu)$ be a $\sigma$-finite measure space and let $L^{0}(\mu)$ denote the space of (equivalence classes) of real measurable functions. Moreover let a function $\psi:[0, \infty) \times T \rightarrow$ $[0, \infty)$ be such that

(1) $\psi(r, \cdot)$ is $\Sigma$-measurable for every $r$,

(2a) $\psi(r, t)=0$ if and only if $r=0$,

(2b) $\lim _{r \rightarrow 0^{+}} \psi(r, t)=0$ for every $t \in T$,

(2c) $\psi(\cdot, t)$ is convex for every $t \in T$,

(2d) $\lim _{r \rightarrow \infty} \frac{\psi(r, t)}{r}=\infty$ for every $t \in T$.

It is well known (see [24, Chapter 2]) that the conjugate function $\psi^{*}(r, t)=$ $\sup _{u \geqslant 0}(u r-\psi(u, t))$ satisfies conditions (1) and (2) and moreover $\psi^{* *}=\psi$. Let $L^{\psi}(\mu)$ be the Musielak-Orlicz space generated by $\psi$, i.e.,

$$
L^{\psi}(\mu)=\left\{f \in L^{0}(\mu): \exists_{s>0} I_{\psi}(f)=\int_{T} \psi(s|f(t)|, t) d \mu<\infty\right\}
$$

A Musielak-Orlicz space $L^{\psi}(\mu)$ is a Banach lattice under the standard $\mu$-almost everywhere pointwise order and under the Luxemburg norm $\|f\|=\inf \{s>0$ : $\left.I_{\psi}(f / s) \leqslant 1\right\}$. If $\psi\left(r, t_{1}\right)=\psi\left(r, t_{2}\right)$ for all $t_{1}, t_{2} \in T$, then $L^{\psi}(\mu)$ becomes an Orlicz space (the reader interested in details about this type of spaces is referred to $[20,21])$. In the sequel the notation $1_{A}$ will denote the characteristic function of a set $A$. Musielak-Orlicz spaces form an essential generalization of Orlicz spaces. If $\lambda$ is the Lebesgue measure on the unit interval, $p, q \in(1, \infty) \backslash\{2\}$ are distinct, then $L^{\psi}(\lambda)$ generated by $\psi(r, t)=r^{p} 1_{\left[0, \frac{1}{2}\right]}(t)+r^{q} 1_{\left[\frac{1}{2}, 1\right]}(t)$ is isomorphic (as a Banach space) to none Orlicz space (see [14] for details).

Every Musielak-Orlicz space has the Levi property (i.e., norm bounded increasing nets of positive elements have a supremum) and the ideal $L^{\psi}(\mu)_{A}=\left\{f \in L^{\psi}(\mu)\right.$ : $\left.|f| \geqslant f_{\alpha} \downarrow 0 \Rightarrow\left\|f_{\alpha}\right\| \rightarrow 0\right\}$ of elements with order continuous norm is super order dense in $L^{\psi}(\mu)$ (see [28, Theorem 1.1, Corollary 1.4]). Repeating the proof of [22, Theorem 1] (or using results from [24, Section 13]) we obtain $L^{\psi}(\mu)_{n}=L^{\psi^{*}}(\mu)$, i.e., the space of order continuous functionals on $L^{\psi}(\mu)$ can be identified with $L^{\psi^{*}}(\mu)$.

We restrict our considerations to functions $\psi$ satisfying the condition

$$
\left(\psi_{\infty}\right) \quad \frac{\psi(2 r, t)}{\psi(r, t)} \underset{r \rightarrow \infty}{\longrightarrow} \infty \text { uniformly with respect to } t \in T \text {. }
$$


The assumption $\left(\psi_{\infty}\right)$ seems to be restrictive but there exist a lot of functions satisfying it. Indeed, suppose that $p: T \rightarrow[1, \infty)$ is measurable, a function $\varphi(r)=$ $\varphi(r, t)$ satisfies conditions (2) and $\frac{\varphi(2 r)}{\varphi(r)} \underset{r \rightarrow \infty}{\longrightarrow} \infty$ (consider for instance $\varphi(r)=$ $\left.\mathrm{e}^{r}-1\right)$. Defining $\psi(r, t)=\varphi(r p(t)) / \varphi\left(p(t)^{2}\right)$ we get a required function. Moreover, if $\mu$ is finite and $p(t)$ is unbounded, then $p(t) \in L^{\psi}(\mu) \backslash L^{\infty}(\mu)$.

Modifying ideas used by Leung in [19] we can prove the following result.

Proposition 4.2 If $\psi$ satisfies condition $\left(\psi_{\infty}\right)$ and $1_{T} \in L^{\psi}(\mu)$, then $L^{\psi}(\mu) \in$ (DPSP).

Proof Let $0 \leqslant f_{n} \rightarrow 0$ in the weak* topology and suppose $\left\|f_{n}\right\| \nrightarrow 0$. Passing to a subsequence, if necessary, and using Theorem 2.2 we can assume $f_{n}\left(x_{n}\right)>2 \eta>0$ for some norm bounded disjoint sequence $\left(x_{n}\right) \subset L^{\psi}(\mu)_{+}$. Denote $M=\sup _{n}\left\|x_{n}\right\|$ and put $A_{n, k}=\left\{t: x_{n}(t)>k\right\}$. Since $1_{T} \in L^{\psi}(\mu)$ then $2 \eta<f_{n}\left(x_{n} 1_{A_{n, k}}\right)+k f_{n}\left(1_{T}\right)$ and the weak* convergence of $\left(f_{n}\right)$ allows us to find a subsequence $\left(n_{k}\right)$ such that $\eta<f_{n_{k}}\left(x_{n_{k}} 1_{A_{n_{k}, k}}\right)$. Define functions $y_{k}=\frac{1}{2(M+1)} x_{n_{k}} 1_{A_{n_{k}, k}}$. For an arbitrary $\varepsilon>0$ there exists $r_{0}$ having a property $\psi\left(\frac{1}{2} r, t\right)<\varepsilon \psi(r, t)$ for all $t \in T$ and $r \geqslant r_{0}$. Taking numbers $k>r_{0}$ we obtain $\int_{T} \psi\left(y_{k}(t), t\right) d \mu \leqslant \varepsilon \int_{T} \psi\left(\frac{1}{M+1} x_{n_{k}} 1_{A_{n_{k}, k}}(t), t\right) d \mu \leqslant \varepsilon$, i.e., $\int_{T} \psi\left(y_{k}(t), t\right) d \mu \rightarrow 0$. Thus $\sum_{j=1}^{\infty} \int_{T} \psi\left(y_{k_{j}}(t), t\right) d \mu<\infty$ for some subsequence $\left(k_{j}\right)$. Since $y_{k}$ are disjoint then $\int_{T} \psi\left(\bigvee_{j=1}^{m} y_{k_{j}}(t), t\right) d \mu=\sum_{j=1}^{m} \int_{T} \psi\left(y_{k_{j}}(t)\right.$, $t) d \mu$, and so the sequence $\left(\bigvee_{j=1}^{m} y_{k_{j}}\right)$ is norm bounded in $L^{\psi}(\mu)$. According to the Levi property $\sup _{j} y_{k_{j}}=y$ exists in $L^{\psi}(\mu)$ but $f_{n_{k_{j}}}(y) \geqslant f_{n_{k_{j}}}\left(y_{n_{k_{j}}}\right)>$ $\eta(2(M+1))^{-1}>0$, a contradiction because $f_{n_{k_{j}}} \rightarrow 0$ in the weak* topology.

Let us note that if $L^{\psi}(\mu)_{n s}$ denotes the space of continuous singular functionals on $L^{\psi}(\mu)$ (i.e., the orthogonal complement of $\left.L^{\psi}(\mu)_{n}\right)$, then $L^{\psi}(\mu)_{n s}=\{f \in$ $\left.\left(L^{\psi}(\mu)\right)^{*}: f\left(L^{\psi}(\mu)_{A}\right)=\{0\}\right\}$. Indeed, as we said before $L^{\psi}(\mu)_{A}$ is order dense in $L^{\psi}(\mu)$, and so a functional $f$ vanishing on $L^{\psi}(\mu)_{A}$ has the trivial carrier. Using [39, Theorem 24.2] we obtain $f \in L^{\psi}(\mu)_{n s}$. Conversely, if $f \in L^{\psi}(\mu)_{n s}$ and additionally the carrier of $f$ reduces to zero, then the absolute kernel $N_{f}$ of $f$ is order dense. Therefore every $0 \leqslant x \in L^{\psi}(\mu)_{A}$ is a supremum of an increasing net of positive elements $\left(x_{\alpha}\right) \subset N_{f}$. Since $L^{\psi}(\mu)_{A}$ is an ideal, then $x_{\alpha} \in L^{\psi}(\mu)_{A}$ and $x_{\alpha} \rightarrow x$ in norm. We obtained that $f$ vanishes on $L^{\psi}(\mu)_{A}$. Now consider a functional $f \in$ $L^{\psi}(\mu)_{n s}$ whose carrier is nonzero. By [39, Theorem 24.3] $|f|=\sup _{\alpha} f_{\alpha}$ for some increasing net $\left(f_{\alpha}\right)$ of singular functionals with trivial carriers. There holds $|f|(x)=$ $\sup _{\alpha} f_{\alpha}(x)$ for $0 \leqslant x \in L^{\psi}(\mu)$, and so $f\left(L^{\psi}(\mu)_{A}\right)=0$ because $f_{\alpha}\left(L^{\psi}(\mu)_{A}\right)=\{0\}$.

As a consequence of our previous considerations we obtain that $L^{\psi}(\mu)_{n s}$ is order isomorphic and isometric to $\left(L^{\psi}(\mu) / L^{\psi}(\mu)_{A}\right)^{*}$ but the quotient $L^{\psi}(\mu) / L^{\psi}(\mu)_{A}$ is an AM-space (see [29] the proof of Theorem 10). Finally $L^{\psi}(\mu)_{n s}$ is an AL-space, and we get the following result: if $L^{\psi}(\mu) \in$ (DPSP), then all even duals of $L^{\psi}(\mu)$ have the dual positive Schur property. Indeed, Musielak-Orlicz spaces $L^{\psi}(\mu)$ having the dual positive Schur property are perfect because $L^{\psi}(\mu)_{n}=L^{\psi^{*}}(\mu)$ has an order continuous norm as a band in the space $\left(L^{\psi}(\mu)\right)^{*} \in(\mathrm{PSP})$. Therefore $\left(L^{\psi^{*}}(\mu)\right)^{*}=L^{\psi^{*}}(\mu)_{n}=L^{\psi^{* *}}(\mu)=L^{\psi}(\mu)$. Hence even duals of $L^{\psi}(\mu)$ are of the 
form $L^{\psi}(\mu) \oplus C(K)$ for some compact space $K$. Arguments applied above allow us to formulate more general result.

If a Banach lattice $E \in$ (DPSP) is perfect and $E$ is a semi-M-space (i.e., $E_{n s}$ is order isomorphic and isometric to an AL-space ), then even duals of $E$ have the dual positive Schur property.

Sometimes a property $E^{*} \in(\mathrm{PSP})$ is sufficient for $E \in$ (DPSP).

Proposition 4.3 For a Musielak-Orlicz space $L^{\psi}(\mu)$ the following statements are equivalent.

(a) $L^{\psi^{*}}(\mu) \in$ (PSP).

(b) $L^{\psi}(\mu) \in$ (DPSP).

Proof $(\mathrm{a}) \Rightarrow$ (b) The positive Schur property implies that $L^{\psi^{*}}(\mu)$ is weakly sequentially complete, and so also the dual $\left(L^{\psi}(\mu)\right)^{*}$ is weakly sequentially complete because $\left(L^{\psi}(\mu)^{*}=L^{\psi^{*}}(\mu) \oplus L^{1}(\nu)\right.$ for some measure $v$. By [18, Theorem 6] (or [12, Proposition 6.1]) the space $L^{\psi}(\mu)$ has the Grothendieck property and Proposition 2.5 finishes the proof.

(b) $\Rightarrow$ (a) Obvious.

There exist spaces $L^{\psi}(\mu) \in$ (DPSP) generated by functions $\psi$ which do not satisfy the assumption $\left(\psi_{\infty}\right)$. Consider the counting measure $\mu$ on $\mathbb{N}$ and a function $\psi(r, n)=$ $\frac{1}{q_{n}} r^{q_{n}}$ where $1<q_{n} \rightarrow \infty$. Clearly $\frac{\psi(2 r, n)}{\psi(r, n)}=2^{q_{n}}$ and $\psi^{*}(r, n)=\frac{1}{p_{n}} r^{p_{n}}$ where $\frac{1}{p_{n}}+\frac{1}{q_{n}}=1$. Since the numbers $b_{n}=\sup _{r>0} \frac{r\left(\psi^{*}(r, n)\right)^{\prime}}{\psi^{*}(r, n)}$ tend to one (in fact we have $b_{n}=p_{n}$ ), then $L^{\psi^{*}}(\mu) \in(\mathrm{SP})$ by Yamamuro theorem (see [30,36]). Applying Proposition 4.3 we obtain $L^{\psi}(\mu) \in$ (DPSP).

Considering our next example we will need the notion of the Riesz angle, denoted by $\mu_{2}(E)$, which plays an important role in the fixed point theory (see [7]). For a Banach lattice $E$ the Riesz angle is defined by

$$
\mu_{2}(E)=\sup \left\{\left\|x_{1} \vee x_{2}\right\|: 0 \leqslant x_{i},\left\|x_{i}\right\| \leqslant 1, i=1,2\right\}
$$

A behavior of $\mu_{2}(E)$ is related to semi-M-spaces. The following result was proved in [34].

Proposition 4.4 For a Banach lattices $E_{n}, n \in \mathbb{N}$, the following statements are equivalent.

(a) $\left(\oplus E_{n}\right)_{\ell} \infty$ is a semi-M-space.

(b) All $E_{n}$ 's are semi-M-spaces and $\lim _{n \rightarrow \infty} \mu_{2}\left(E_{n}\right)=1$.

Consider the space $E=\left(\oplus \ell_{k}^{q_{k}}\right)_{\ell_{\infty}}$ where $1<q_{k} \rightarrow \infty$. $E$ is a semi-M-space because $\mu_{2}\left(\ell_{k}^{q_{k}}\right)=2^{\frac{1}{q_{k}}}$. According to Proposition 3.1 we have $E_{n}=\left(\oplus \ell_{k}^{p_{k}}\right)_{\ell^{1}}$ (where $\frac{1}{p_{k}}+\frac{1}{q_{k}}=1$ ). Therefore $E_{n} \in(\mathrm{SP})$ (see [30]). Since $E^{*}=E_{n} \oplus L^{1}(v)$ for some measure $v$, then $E^{*} \in(\mathrm{PSP})$. Räbiger showed in [26, Satz 9.7] that $E \in(\mathrm{GP})$. Hence $E \in$ (DPSP) by Proposition 2.5 and also even duals of $E$ have the dual positive Schur property because $E$ is perfect. 
All our examples of Banach lattices with the dual positive Schur property are such that their even duals have this property too. It is natural to ask if there exists a Banach lattice $E \in(\mathrm{DPSP})$ with $E^{* *} \notin$ (DPSP).

We finish the paper presenting an example of a Banach lattice which confirms that the theorem converse to Proposition 2.10 is false. Hernadez in [15] indicated a hereditarily $\ell^{1}$ Orlicz sequence space $\ell^{\varphi}$ (i.e., every infinite dimensional closed subspace of $\ell^{\varphi}$ contains an isomorphic copy of $\ell^{1}$ ) without the Schur property. The space $\ell^{\varphi}$ has order continuous norm because it does not contain $c_{0}$. Put $E=\ell^{\varphi^{*}}$. Since $E^{*}$ is order isomorphic to $\ell^{\varphi} \oplus L^{1}(v)$ then it is weakly sequentially complete. Applying [18, Theorem 6] (or [12, Proposition 5.2]) we obtain $E \in$ (GP). Moreover $E \notin$ (DPSP). Indeed, if there were $E \in$ (DPSP), then $E^{*}$ would have the positive Schur property. Hence $\ell^{\varphi} \in(\mathrm{PSP})$ but discreteness of $\ell^{\varphi}$ implies $\ell^{\varphi} \in$ (SP), a contradiction.

We claim that $E$ can not be positively mapped onto any Banach lattice with order continuous norm. On the contrary, suppose that there exists a Banach lattice $F$ whose norm is order continuous and $T: E \rightarrow F$ is a positive surjection. Repeating arguments used in the first part of the proof of Proposition 2.12 we obtain $F$ has to be reflexive. By the closed range theorem the adjoint operator $T^{*}: F^{*} \rightarrow E^{*}=\ell^{\varphi} \oplus L^{1}(\nu)$ is a positive isomorphic embedding. Let $P: E^{*} \rightarrow \ell^{\varphi}$ and $Q: E^{*} \rightarrow L^{1}(v)$ be band projections. According to the reflexivity of $F^{*}$ every norm bounded sequence $\left(f_{n}\right) \subset F^{*}$ contains weakly convergent subsequences $\left(f_{n_{k}}^{+}\right),\left(f_{n_{k}}^{-}\right)$. Therefore $\left(Q \circ T^{*}\left(f_{n_{k}}^{+}\right)\right),\left(Q \circ T^{*}\left(f_{n_{k}}^{-}\right)\right)$ are weakly convergent positive sequences in $L^{1}(v)$, and so they are norm convergent because $L^{1}(v) \in$ (PSP). We have just shown that $Q \circ T^{*}$ is compact.

The subspace $\left(P \circ T^{*}\right)\left(F^{*}\right)$ is closed in $\ell^{\varphi}$ and it has to be finite dimensional because $\left(P \circ T^{*}\right)\left(F^{*}\right)$ is reflexive as a continuous image of a reflexive space and $\ell^{\varphi}$ is hereditarily $\ell^{1}$. Hence $P \circ T^{*}$ is compact. Finally $T^{*}$ is compact being the sum of two compact operators which contradicts the fact that $T^{*}$ is an isomorphism.

Open Access This article is distributed under the terms of the Creative Commons Attribution License which permits any use, distribution, and reproduction in any medium, provided the original author(s) and the source are credited.

\section{References}

1. Albiac, F., Kalton, N.J.: Topics in Banach Space Theory. In: Graduate Texts in Math., vol. 233. Springer, Berlin (2006)

2. Aliprantis, C., Burkinshaw, O.: Locally Solid Riesz Spaces with Applications to Economics. Mathematical Surveys and Monographs, vol. 105, American Mathematical Society (2003)

3. Aliprantis, C., Burkinshaw, O.: Positive Operators. Springer, Berlin (2006)

4. Aqzzouz, B., Elbour, A.: The Schur property of Banach lattices and the compactness of weakly compact operators. Math. Proc. R. Ir. Acad. 110A, 15-25 (2010)

5. Aqzzouz, B., Elbour, A.: Some characterizations of almost Dunford-Pettis operators and applications. Positivity 15, 369-380 (2011)

6. Aqzzouz, B., Elbour, A., Wickstead, A.W.: Positive almost Dunford-Pettis operators and their duality. Positivity 15, 185-197 (2011)

7. Borwein, J.M., Sims, B.: Nonexpensive mappings on Banach lattices and related topics. Houston J. Math. 10, 339-356 (1984)

8. Chen, Z.L., Wickstead, A.W.: Relative weak compactness of solid hulls in Banach lattices. Indag. Mathem. N.S. 9, 187-196 (1998) 
9. Chen, Z.L., Wickstead, A.W.: Some applications of Rademacher sequences in Banach lattices. Positivity 2, 171-191 (1998)

10. Chen, Z.L., Wickstead, A.W.: L-weakly and M-weakly compact operators. Indag. Mathem. N.S. 10, 321-336 (1999)

11. Dodds, P.G., Fremlin, D.H.: Compact operators in Banach lattices. Israel Math. J. 34, 287-320 (1979)

12. Granero, A.S.: The Grothendieck property in the class of Orlicz-type modular spaces, property in the class of Orlicztype modular spaces. http://www.mat.ucm.es/ granero/Investigacion/Grothendieck

13. Groenewegen, G., Meyer-Nieberg, P.: An elementary and unified approach to disjoint sequence theorems. Indag. Math. 48, 313-317 (1986)

14. Hernandez, F.L., Ruiz, C.: On Musielak-Orlicz spaces isomorphic to Orlicz spaces. Comment. Math. Prace Mat. 32, 55-60 (1992)

15. Hernadez, F.L.: Private communication

16. Kamińska, A., Mastyło, M.: The Schur and (weak) Dunford-Pettis properties in Banach lattices. J. Aust. Math. Soc. 73, 251-278 (2002)

17. Kantorovich, L.V., Akilov, G.P.: Functional Analysis. Nauka, Moscow (1984) (in Russian)

18. Leung, D.H.: Weak* convergence in higher duals of Orlicz spaces. Proc. Am. Math. Soc. 103, 797-800 (1988)

19. Leung, D.H.: On the weak Dunford-Pettis property. Arch. Math. 52, 363-364 (1989)

20. Lindenstaruss, J., Tzafriri, L.: Classical Banach Spaces I. Sequence Spaces. Springer, Berlin (1977)

21. Lindenstaruss, J., Tzafriri, L.: Classical Banach Spaces II. Function Spaces. Springer, Berlin (1979)

22. Maligranda, L., Wnuk, W.: Landau type theorem for Orlicz spaces. Math. Z. 208, 57-64 (1991)

23. Meyer-Nieberg, P.: Banach Lattices. Springer, Berlin (1991)

24. Musielak, J.: Orlicz Spaces and Modular Spaces. Lecture Notes in Math, vol. 1034. Springer, Berlin (1983)

25. Räbiger, F.: Lower and upper 2-estimates for order bounded sequences and Dunford-Pettis operators between certain classes of Banach lattices. In: Proceedings the University of Texas at Austin, 1987/1989, Lecture Notes in Math, vol. 1470, pp. 159-170. Springer, Berlin (1991)

26. Räbiger, F.: Beiträge zur Strukturtheorie der Grothendieck-Räume. Sitzungsberichte der Heidelberger Akademie der Wissenschsften. Math.-Naturwiss. Klasse, Jahrgang, 4. Abh., pp. 85-158. Springer, Berlin, Heidelberg, New York, Tokyo (1985)

27. Wilansky, A.: Modern Methods in Topological Vector Spaces. McGraw-Hill, New York (1978)

28. Wnuk, W.: Representations of Orlicz lattices. Diss. Math. 235, 1-62 (1984)

29. Wnuk, W.: On the order-topological properties of the quotient spaces $L / L_{A}$. Studia Math. 79, 139-149 (1984)

30. Wnuk, W.: Banach lattices with properties of the Schur type: a survey. Conf. Sem. Mat. Univ. Bari 249, 1-25 (1993)

31. Wnuk, W.: Banach lattices with the weak Dunford-Pettis property. Atti Sem. Mat. Fis. Univ. Modena 42, 227-236 (1994)

32. Wnuk, W.: Banach Lattices with Order Continuous Norms. Polish Scientific Publishers PWN, Warszawa (1999)

33. Wnuk, W.: The strong Schur property in Banach lattices. Positivity 13, 435-441 (2009)

34. Wnuk, W.: Remarks concerning M-constants in Banach lattices, in preparation

35. Wójtowicz, M.: A Banach-lattice version of the Josefson-Nissenzweig theorem. Indag. Mathem. N.S., 18, 479-484 (2007)

36. Yamamuro, S.: Modulared sequence spaces. J. Fac. Sci. Hokkaido Univ. 13, 50-58 (1954)

37. Zaanen, A.C.: Integration. North Holland, Amsterdam (1967)

38. Zaanen, A.C.: Riesz Spaces II. North Holland Publishing Company, Amsterdam (1983)

39. Zaanen, A.C.: Introduction to Operator Theory in Riesz Spaces. Springer, Berlin (1997) 